Ann. Zootech., I967, 16 (3), 235-245.

\title{
INFLUENCE DU MILIEU THERMIQUE SUR L'ÉVOLUTION DE LA TEMPÉRATURE RECTALE ET DE LA GLYCÉMIE CHEZ LE PORCELET NOUVEAU-NÉ
}

\author{
H. de la PORTE des VAUX, A. AUMAITRE \\ avec la collaboration technique de R. Deniel et Huguette Dewllf \\ Station de Recherches sur l'Élevage des Porcs, \\ Centre national de Recherches zootechniques, 78-Jouy-en-Josas, \\ Institut national de la Recherche agronomique
}

\section{SOMMAIRE}

L'effet de la température du local de mise bas a été étudié sur 12 portées de porcelets nés à $11^{\circ}$, $18^{\circ}$ et $28^{\circ}$ respectivement. La vitesse de croissance des animaux jusqu'à 5 jours est faible à $11^{\circ}$, alors qu'elle est maximum à $28^{\circ}$. C'est pour la température la plus basse que la mortalité est la plus élevée (42 p. 100). La nécessité du chauffage des locaux de maternité est soulignée.

On constate une chute brutale de la température rectale suivant immédiatement la naissance, (de 39 à $34,2^{\circ}$ ) ; l'intensité et la durée de cette chute sont d'autant plus fortes que la température est plus faible.

Parallèlement à la baisse de la température centrale, la glycémie présente également un minimum variable suivant le milieu et d'autant plus tardif que la température est plus faible.

Le dosage spécifique des constituants glucidiques sanguins montre le rôle particulier des réserves en fructose et en glucose dans la thermogenèse du porcelet nouveau-né avant la première alimentation.

\section{INTRODUC'TION}

L'effort d'amélioration des conditions d'élevage des animaux domestiques a conduit à souligner l'importance de l'alimentation, mais aussi l'influence parfois déterminante des conditions de milieu (habitat, température, hygrométrie...) sur la croissance des jeunes animaux.

Le Porc est sensible aux variations climatiques (HopkINs, I928; WALLACH et al., I948 ; LEROY, I 953 ; JENSEN, I964), d'autant plus qu'il est plus jeune (MORILL, I952). On observe ainsi dans les quelques heures qui suivent la naissance un pourcentage élevé de mortalité parmi les animaux nés ou maintenus dans un milieu à basse température (NEWLAND et al., I952; LUCAS, I954). 
D'autre part, on constate que la croissance des animaux est limitée lorsqu'ils sont élevés à basse température (MCLAGAN et Thomson, I950 ; Runcis, I956), alors qu'une augmentation de la température ambiante entraîne une amélioration du gain de poids et une diminution de la mortalité (NEWLAND et al., I952; Zukov, I952).

La naissance s'accompagne d'une diminution brusque de la température corporelle, variable suivant le degré de maturité atteint par la thermorégulation (KAYSER, I964). Chez le porcelet, cette maturité n'est pas complétement développée (MouNT, I959) et le nouveau-né est capable de lutter contre le froid pendant une durée limitée à quelques heures seulement par ses réserves énergétiques propres (MCCANCE et WIDDowson, I959). Celles-ci, d'origine glucidique (NEWLAND et al., I952 ; ELNEIL, et McCance, I965; Curtis et al., ig66) ou lipidiques (Hahn et Koldovsky, r96r $a$ et $b$; NovaK et al., I965; MCCANCE et WIDDOWson, r959), sont mobilisées par l'intervention de nombreux mécanismes physiologiques mis en évidence par Holub et al. (I957), Mount (I959 et ig60), Ingram et Slebodzinski (I965).

Cependant, les phénomènes physiques et chimiques de la thermorégulation répondant aux variations brusques et importantes de la température du local de naissance sont encore insuffisamment connus. Les variations de température centrale ne sont pas observées par tous les auteurs (CURTIS et al., I966), et les variations de la glycémie ne concernent souvent que les sucres réducteurs totaux tandis que les teneurs exactes en chaque composant glucidique sont rarement rapportées.

En vue d'étudier plus précisément cette adaptation de la température corporelle du porcelet nouveau-né au milieu ambiant, nous avons mesuré l'évolution de la température rectale sur un nombre important d'animaux nés dans des milieux différents. Nous avons cherché en outre à relier cette adaptation aux variations de la glycémie en déterminant les taux de sucres réducteurs totaux, de glucose vrai et de fructose du sang jusqu'à l'âge de 5 jours.

\section{MATÉRIEL ET MÉTHODHS}

\section{Animaux}

I 2 truies de race Large White appartenant à l'élevage expérimental du C.N. R. Z. ont été utilisées et affectées au hasard aux trois traitements suivants :

Groupe I : Local maintenu à I $^{\circ}$ pendant la durée de l'expérience

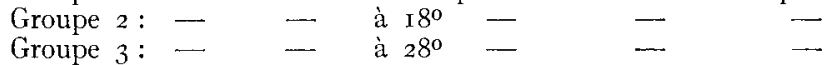

Chaque truie était placée dans le local conditionné à la température désirée quelques jours avant la date prévue de la mise bas. Les porcelets étaient laissés sous la mère, placée en cage de mise bas, et tétaient librement ; au total IOI porcelets ont été utilisés, représentant 4 portées par traitement.

\section{Mesures et dosages effectués}

Les animaux ont été pesés dès la naissance, puis aux âges de $60 \mathrm{~h}$ et $\mathrm{I} 20 \mathrm{~h}$.

La température rectale a été mesurée au $\mathbf{I}$ /Io de degré centigrade au moyen d'un téléthermomètre à thermistance ( $\left.{ }^{1}\right)$ muni de sondes souples. La mesure a été réalisée à chaque fois sur tous les animaux de la portée, toutes les 5 minutes de la naissance à la première demi-heure, puis aux âges exacts de $2,4,8,16,32,60$ et 120 heures.

(1) Yellow Spring Instruments. 
Le dosage des sucres dans le sang a été effectué sur chaque porcelet de la portée à partir de 2 heures d'âge. Les prélèvements ont été réalisés par section franche de la queue sur des animaux héparinés à la naissance (500 $\gamma / \mathrm{kg}$ de poids vif). Le sang était recueilli dans un tube contenant des cristaux d'oxalate de potassium et de fluorure de sodium (WATSON, I962 ; MARKS et Lloyd, I963; LOISELEUR, I963).

Après défécation du sang par addition de sulfate de zinc à $5 \mathrm{p}$. Ioo et de baryte $0,3 \mathrm{~N}$ environ, les sucres réducteurs ont été dosés par la méthode de SomoGyi (I945), le glucose vrai a été dosé spécifiquement après oxydation par la glucose oxydase et colorimétrie en présence d'orthodianisidine selon HUGUETT et NIXON (1957) et le fructose par la méthode colorimétrique de RoE (I934).

\section{Calculs statistiques}

Les valeurs moyennes des températures rectales, ainsi que des taux sanguins en sucres réducteurs, glucose et fructose, ont été comparées en fonction de l'âge à l'aide du test de comparaison multiple des moyennes (DUNCAN, 1955).

La comparaison des valeurs moyennes du poids et du gain de poids a été effect uée par analyse de variance après correction des données par régression linéaire en fonction du poids à la naissance (Legault et Canonge, 1965).

\section{RÉSULTATS}

\section{Vitesses de croissance}

Les gains de poids observés pour les différents groupes au cours des 2 périodes, de la naissance à $60 \mathrm{~h}$, et de la naissance à $\mathrm{I} 20 \mathrm{~h}$ ( 5 jours), sont rapportés au tableau I. Les valeurs observées sont toutes significativement différentes : les animaux nés dans un milieu à température élevée ont une vitesse de croissance plus rapide surtout au cours de la première période de la vie.

Les pertes d'animaux par écrasement, ou par mort naturelle, sont beaucoup plus importantes aux faibles températures (tabl. I).

\section{TABLEAU I}

Gain de poids et mortalité des porcelets en fonction de la température du milieu

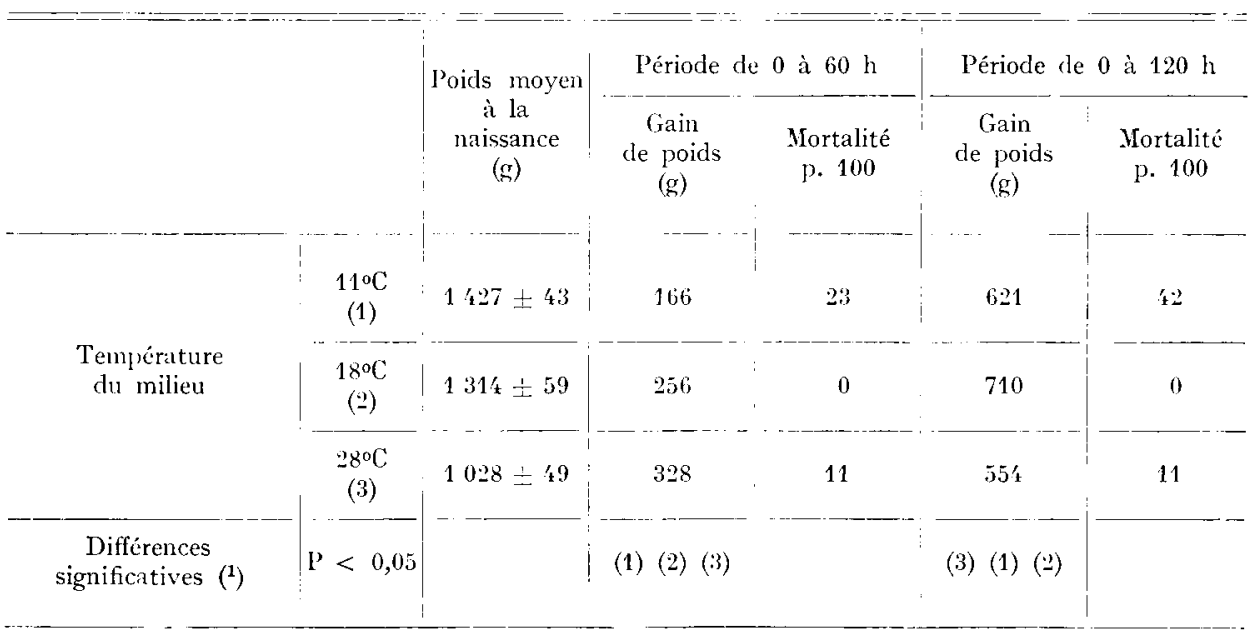

(1) Les parenthèses représentent les différences significatives suivant le milieu.

Annales de Zootechnie. - I 967 . 


\section{Variations de la température rectale}

Nous avons considéré deux phases distinctes au cours desquelles les variations de température rectale du nouveau-né sont mesurées (fig. I).

a) Evolution après la naissance.

Dans chacun des groupes, on observe une chute de température rectale immédiatement après la naissance (fig. I).
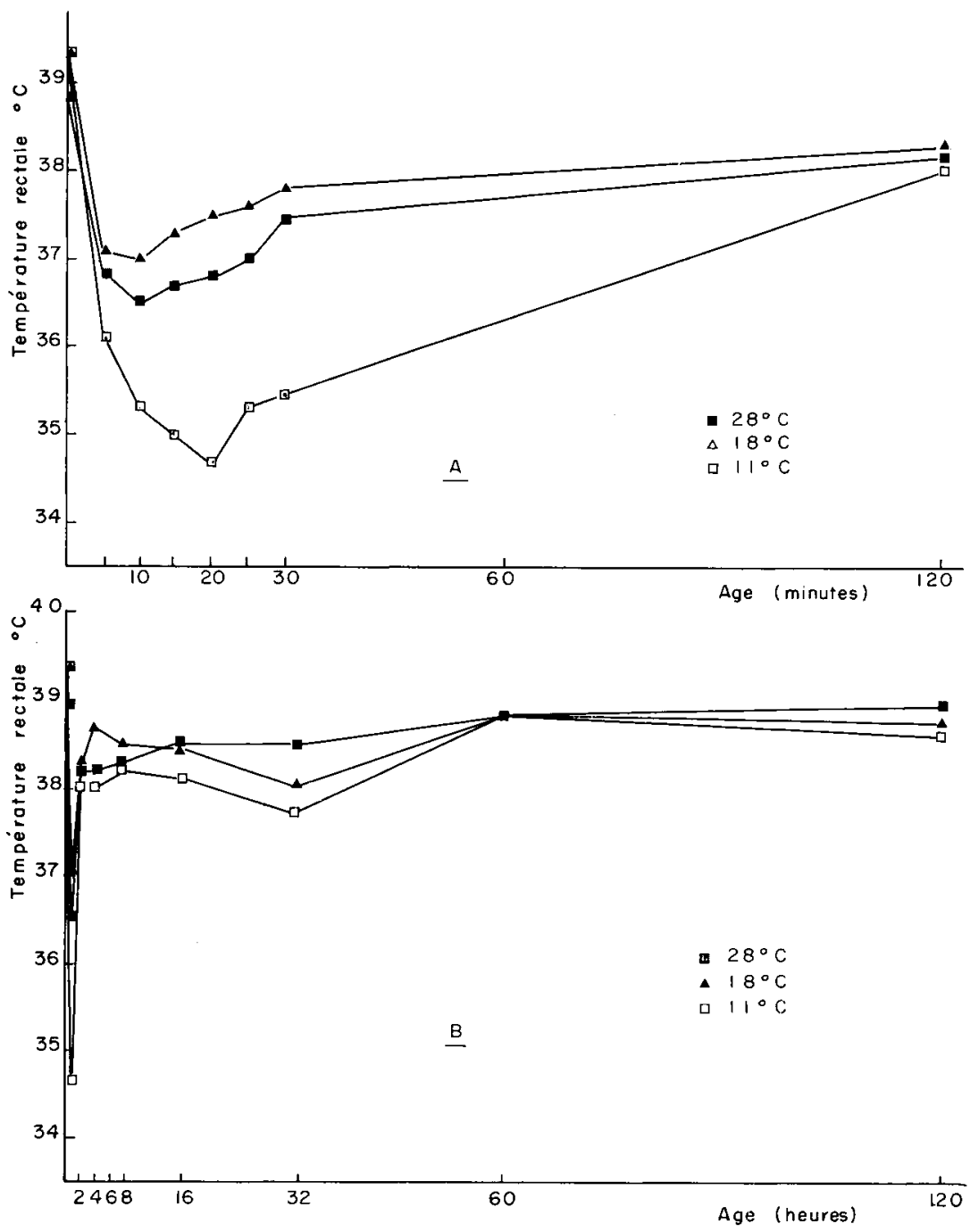

FIG. 1. - Évolution de la température reclale du porcelet après la naissanie en fonction de la température du local de mise-bas

A - De la naissance à 2 h d'âge

B - De la naissance à i 20 heures 
D'une façon générale, la température rectale passe par un minimum qui est atteint plus tôt lorsque la température ambiante est élevée (tab1. 2). La valeur absolue de ce minimum est proportionnelle à la température du milieu ambiant. C'est pour les animaux nés dans un milieu froid $\left(\mathrm{II}^{\circ}\right)$ que $1^{\prime}$ on observe la baisse maximum ( $5^{\circ}$ en 20 minutes).

D'autre part, cette baisse de température est d'autant plus faible que le poids à la naissance est plus élevé. Il existe, en effet, un coefficient de corrélation négatif très élevé entre la valeur de la chute de température rectale des animaux et le poids à la naissance (tabl. 2).

\section{TABLEAU 2}

Valeurs moyennes de la chute de température rectale suivant le milieu; coefficient de corrélation entre la valeur de cette chute et le poids à la naissance

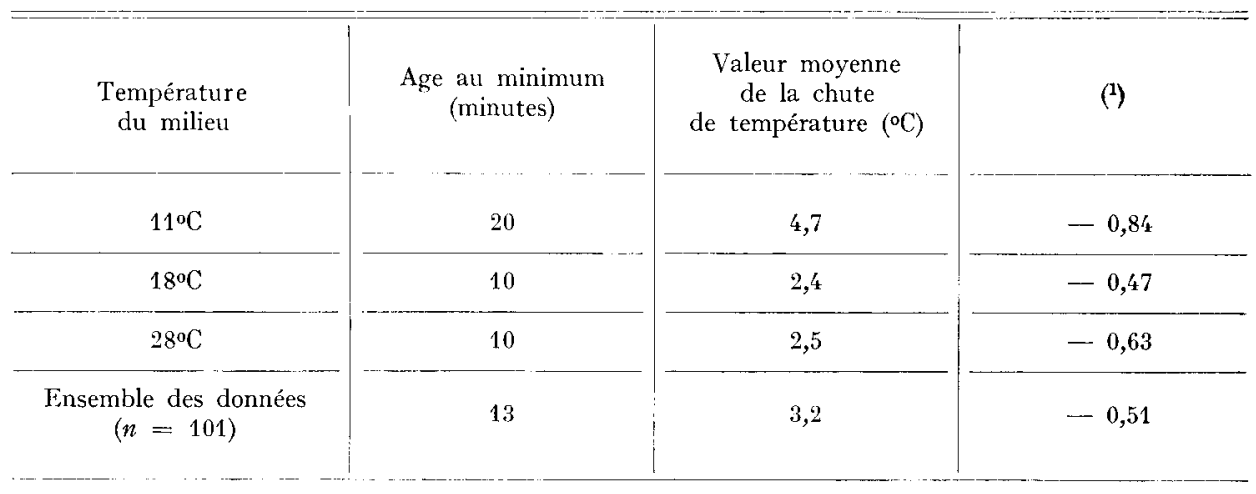

(1) $\mathrm{P}<0,01$.

Le temps nécessaire à la remontée de la température à une valeur normale varie également avec les traitements : il est beaucoup plus long aux basses températures ( 2 heures à II ${ }^{\circ} \mathrm{C}$ contre environ 30 minutes à $28^{\circ} \mathrm{C}$ ).

b) Évolution entre 2 heures d'âge et 120 heures.

L'amplitude de variation de la température est faible mais on observe toujours une température élevée chez les animaux maintenus dans un milieu plus chaud. Après 48 heures, les variations sont de plus en plus limitées.

c) Relation entre le poids et la température rectale.

Les coefficients de corrélation calculés entre le poids et la température rectale sont en général faibles et non significatifs $(r=+0,20$ à la naissance ; $r=-0,09$ à 60 heures) ; ils montrent qu'il n'existe pas de liaison linéaire entre les dewx valeurs.

\section{Variation du taux de sucres dans le sang}

D'une façon générale, l'évolution de la glycémie en fonction de l'âge ( 2 heures à I 20 heures) variebeaucoup moins d'un milieu à l'autre que la température corporelle. 
a) Sucres réducteurs totaux.

Les valeurs moyennes ainsi que l'évolution du taux de sucres réducteurs totaux en fonction de l'âge et de la température sont présentées au tableau 3 et à la figure 2 . Pour les animaux des différents groupes, les glycémies initiales sont comparables. Par contre, la chute du taux de sucres réducteurs sanguins observée ensuite est d'autant plus rapide et plus importante que la température ambiante est plus faible. Cette baisse atteint I $8 \mathrm{I} \mathrm{mg} / 1$ à 60 heures, I 7 I mg/1 à 32 heures et II $7 \mathrm{mg} / 1$ à I 6 heures aux températures respectives de $I I, x 8$ et $28^{\circ}$.

\section{TABLEAU 3}

Évolution des taux de sucres réducteurs, de glucose et de fructose dans le sang en fonction de l'âge et de la température. Signification des différences observées

\begin{tabular}{|c|c|c|c|c|c|c|c|}
\hline & & & \multicolumn{4}{|c|}{ Age des porcelets en heures } & \multirow{2}{*}{$\begin{array}{l}\text { Signifi- } \\
\text { cation (1) }\end{array}$} \\
\hline & & & $2(a)$ & $(b)$ & $32(c)$ & $120(d)$ & \\
\hline \multirow{4}{*}{$\begin{array}{l}\text { Sucres } \\
\text { réducteurs } \\
(\mathrm{g} / \mathrm{l})\end{array}$} & \multirow{3}{*}{$\begin{array}{c}\text { Tempé- } \\
\text { rature } \\
\text { du milieu }\end{array}$} & $11^{\circ} \mathrm{C}(1)$ & 1,190 & 1,102 & $--\longrightarrow--$ & 1,310 & $(a)(b)(c)(d)$ \\
\hline & & $18^{\circ} \mathrm{C}(2)$ & 1,280 & 1.170 & 1,030 & 1,218 & $a(b)(c)(d)$ \\
\hline & & $28^{\circ} \mathrm{C}(3)$ & 1,106 & 1,056 & 1,050 & 1,223 & (a) b, c, (d) \\
\hline & \multicolumn{2}{|c|}{ Signification $\left({ }^{1}\right)$} & (1) (2) (3) & (1) (2) (3) & $(1), 2,3$ & $(1), 2,3$ & \\
\hline \multirow{4}{*}{$\begin{array}{l}\text { Glucose } \\
(\mathrm{g} / \mathrm{l})\end{array}$} & \multirow{3}{*}{$\begin{array}{l}\text { 'Tempé- } \\
\text { rature }\end{array}$} & $11^{\circ} \mathrm{C}$ & 0,869 & 0,802 & 0,650 & 0,930 & $(a)(b)(c)(d)$ \\
\hline & & $18^{\circ} \mathrm{C}$ & 0,799 & $0,81 \bar{j}$ & 0,729 & 0,981 & $a, b,(c)(d)$ \\
\hline & & $28^{\circ} \mathrm{C}$ & 0,683 & $0,6 \pm 0$ & 0,672 & 0,799 & $a(b) c(d)$ \\
\hline & \multicolumn{2}{|c|}{ Signification $\left({ }^{\mathbf{1}}\right)$} & (1) (2) (3) & $1,2(3)$ & 1 (2) 3 & (1) (2) (3) & \\
\hline \multirow{4}{*}{$\begin{array}{l}\text { Fructose } \\
\qquad(\mathrm{g} / \mathrm{l})\end{array}$} & \multirow{3}{*}{$\begin{array}{l}\text { 'Tempé- } \\
\text { rature }\end{array}$} & $11^{\circ} \mathrm{C}$ & 0,090 & $0,0^{\prime} 6$ & $0,0 \div 1$ & 0,035 & $(a)(b)(c)(d)$ \\
\hline & & $18^{\circ} \mathrm{C}$ & 0,125 & 0,057 & 0,025 & 0,045 & $(a)(b)(c)(d)$ \\
\hline & & $28^{\circ} \mathrm{C}$ & $0,13^{\prime}$ & $0,0 \div 1$ & $0,0<4$ & 0,58 & (a) $b, c,(d)$ \\
\hline & \multicolumn{2}{|c|}{ Signification $(\mathbf{1})$} & (1) (2) (3) & (1) (2) (3) & $1(2), 3$ & (1) (2) (3) & \\
\hline
\end{tabular}

(I) Les valeurs entre parenthèses sont significativement différentes $\left(\mathrm{P}^{\prime}<0,05\right)$.

b) Glucose.

L'évolution de la glycémie vraie est semblable à celle des sucres réducteurs totaux, ce qui s'explique par le fait que le glucose constitue environ les $2 / 3$ des sucres réducteurs totaux (63 à $7 \mathrm{I}$ p. Ioo). Les valeurs moyennes de la glycémie vraie (glu- 


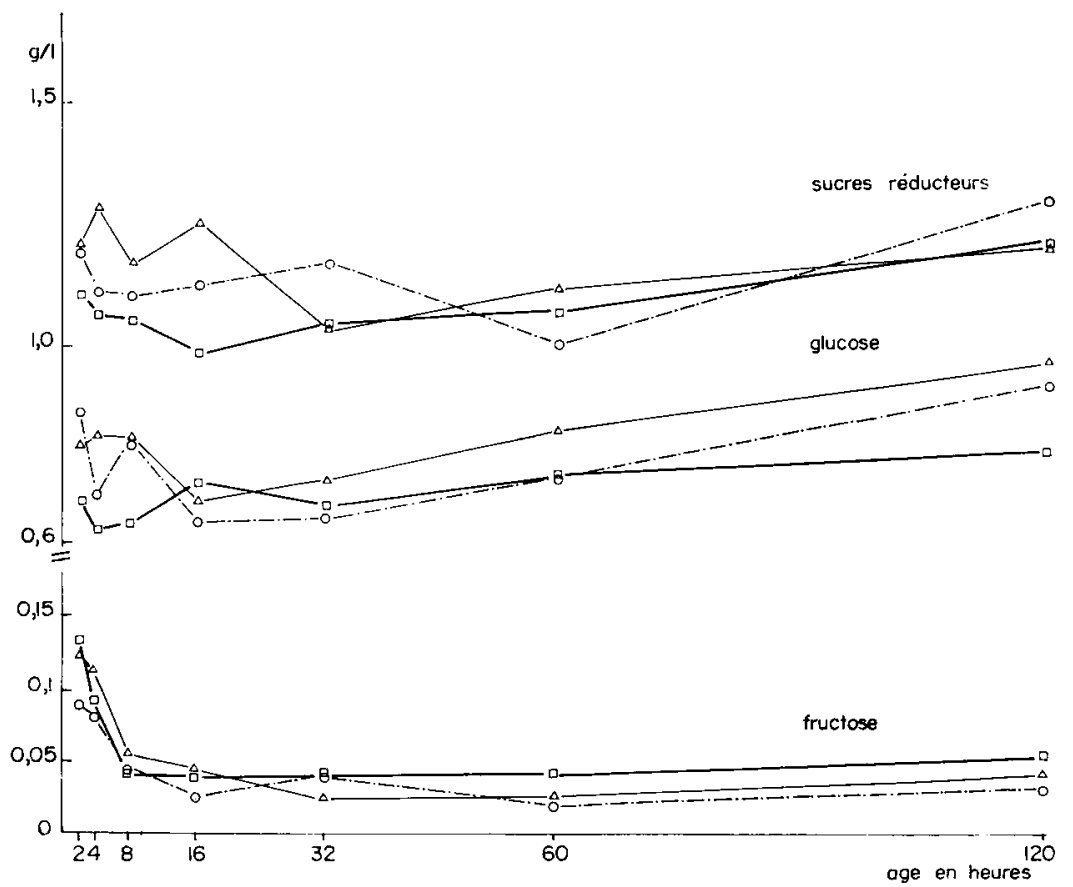

Fig. 2. - Évolution de la glycémie pendant les premiers jours de la vie du porcelet en fonction de la température du local de naissance

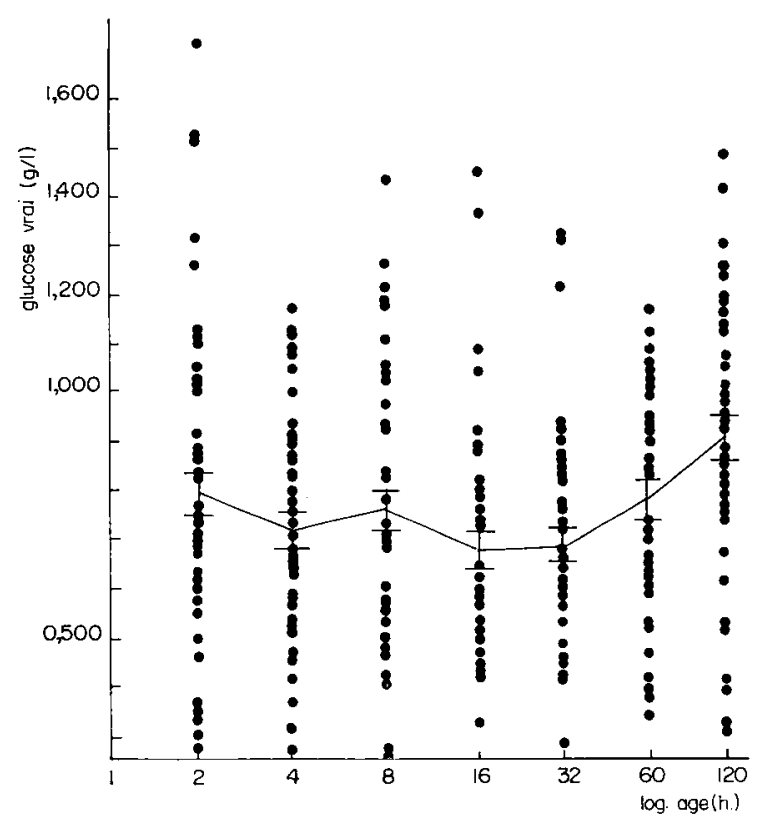

lig. 3. - Valeurs indiaiduelles du taux de glucose vrai en fonction de l'àge du porcelet (Valeur moyenne et intervalle de confiance de la moyenne $\pm s \bar{x}$ ) 
cose sanguin) sont rapportées au tableau 3. On constate une dispersion très importante des valeurs individuelles entre les porcelets, qui se retrouve à tous les âges (fig. 3).

Quelle que soit la température ambiante, on observe cependant une valeur minimum du taux de glucose située dans le même ordre chronologique que celle des sucres réducteurs. Néanmoins, la chute du taux de glucose est plus importante à II $^{\mathbf{0}}$ (2I9 $\mathrm{mg} /$ litre) et en valeur relative moindre à $28^{\circ}(59 \mathrm{mg} /$ litre $)$ que celle du taux de sucres réducteurs, ce qui peut faire penser à une utilisation du glucose pour la thermogénèse aux faibles températures.

\section{c) Fructose.}

Les résultats concernant les valeurs du taux de fructose dans le sang sont rapportés au tableau 3 et à la figure 2 .

L,es valeurs trouvées sont généralement faibles, mais présentent une évolution très caractéristique. Indépendamment de la température du local de mise bas, le taux de fructose diminue de 40 à $60 \mathrm{p}$. roo entre les âges de 2 heures et de 8 heures.

Les taux semblent également présenter une valeur minimum, puis une légère remontée avec l'âge mais, compte tenu des quantités très faibles retrouvées, environ $40 \mathrm{mg} /$ litre (35 à $55 \mathrm{mg}$ ), celle-ci est assez peu sensible.

\section{DISCUSSION}

I e chauffage du local de naissance entraîne une augmentation significative de la croissance de tous les animaux et du taux de survie. En raison de l'affectation au hasard des truies aux différentes températures, les porcelets placés à I I ${ }^{0}$ étaient significativement plus lourds à la naissance. Bien que ce phénomène ait pu minimiser l'effet propre au milieu, des différences de croissance importantes apparaissent entre les lots. Ces données sont désormais classiques et trouvent une application intéressante dans la pratique du chauffage des locaux d'élevage (WAI, ACH et al., I948; NEWLAND et al., I952; JENSEN, r954).

Le point intéressant concerne les variations physiologiques entraînées par les modifications du milieu. Celles-ci se manifestent en premier lieu sur la température corporelle : lors du passage du milieu utérin au milieu ambiant, les porcelets présentent une chute de température centrale d'autant plus forte que le milieu est plus froid et que leur poids est plus faible. Nos résultats sont en accord avec ceux observés par NeWLand et al. (I952) ; Mount et al. (I959 et I96I), seuls Curtis et al. (I966) n'ont pas observé de baisse de la température rectale car ils ont utilisé des intervalles de temps trop longs entre les mesures.

Ce phénomène paraît très général chez les mammifères. On le rencontre en effet chez le Rat (DAwEs et Mo'Tr, I959) et également chez 1'Énfant (SMiтH, I959), enfin il a été observé par ALEXANDER et MCCANCE (I958) sur l'Agneau et par Markovic (I.963) sur le Veau nouveau-né. 'Toutefois pour les différentes espèces la durée et l'intensité de la chute de température centrale et rectale varient et pour- 
raient servir de critères pour caractériser le degré de maturité atteint par les mécanismes de la thermorégulation à la naissance (KAYSER, I963).

Dans une certaine mesure le chauffage du local de naissance permet de réduire les effets néfastes dus aux milieux froids et favorise un retour plus rapide à la normale.

Enfin, la forte liaison inverse entre la chute de température rectale et le poids à la naissance des jeunes animaux est intéressante à considérer indépendamment du traitement. Elle peut expliquer que les pertes en animaux à la naissance soient plus importantes pour les races les plus prolifiques dont le poids à la naissance est généralement plus faible.

Par ailleurs la chute de température s'accompagne d'une évolution caractéristique des constituants glucidiques du sang considérés comme principaux agents de la thermogenèse après la naissance et avant la première alimentation, (MCCANCE et Widdowson, I959; SHElley et NEI,IGAN, I966).

Les valeurs individuelles observées tant pour les taux de sucres réducteurs que pour les taux de glucose vrai varient dans de très larges"limites. Nous retrouvons un phénomène identique à celui observé chez l'enfant par FARQUAR, (I954), mais les valeurs sont également dispersées quelque soit l'âge de l'animal.

Les valeurs moyennes de la glycémie (sucres réducteurs et glucose) présentent un minimum d'autant plus tardif que le milieu est plus froid. L'hypoglycémie résulte du fait que la naissance prive le jeune organisme d'apport énergétique (KAYSER, I963) au moment où la thermorégulation entraîne la combustion des réserves glucidiques. De plus l'insuffisance du renouvellement de ces réserves pendant les premières heures de la vie peut également provenir de la très faible teneur du colostrum de la Truie en lactose (SALMON-LEGaGNEUR et GuÉGUEN, I962). Enfin, l'utilisation préférentielle des sucres (fructose, glucose) pour la thermogneèse (MCCANCE et WIDDowsox, 1959) peut expliquer également ces valeurs minimum.

Cependant, la valeur des dépenses énergétiques est très élevée et les réserves glucidiques limitées surtout dans le cas du jê̂ne où des animaux placés en milleux froids. On peut penser à une participation active des tissus corporels, principalement du tissu adipeux, à la thermogenèse ainsi que le montrent les résultats de HAHN et Koldovsky, (I95I $a$ et I963) HaHN et al. (I96r $b$ ) retrouvés chez le porcelet par MCCANCE et WIDDowson (I959); ces données permettraient d'expliquer une remontée de la glycémie avec l'âge qui est généralement observée avant même la première alimentation.

L'évolution du taux de fructose sanguin est très caractéristique, il ne baisse pas de la même façon que le glucose et varie indépendamment de la température. Nos résultats sont en accord avec ceux de Goodwin (I956) et CurTis et al. (I966) en ce qui concerne l'évolution avec l'âge, et avec ceux de KIDDER et al. (I963) pour les valeurs absolues. Le fructose est catabolisé à des fins énergétiques au cours des 8 premières heures de la vie du porcelet, mais étant donné ses quantités relativement faibles, son rôle est forcément très limité, mais différent de celui des autres sucres.

Nos résultats permettent de penser que la thermorégulation s'installe chez le porcelet nouveau-né pendant les deux premières heures de sa vie (Hor,uB et al. I957; Mouxt, I959). Il existe une participation importante des réserves corporelles dans la thermogénèse après la naissance, d'autant plus notable que la température du local de mise-bas est faible. L'animal peut ainsi épuiser ses réserves pour maintenir sa 
température avant la première alimentation, ce qui peut expliquer les pertes nombreuses enregistrées en hiver ou dans les locaux froids dans les conditions pratiques de l'élevage.

Ręu pour publication en mai 1967.

\section{SUMMARY}

THE EFFECT OF ENVIRONMENTAL TEMPERATURE ON RECTAL TEMPERATURE

AND BLOOD SUGAR LEVEL OF THE NEW-BORN PIG

This investigation was carried out on 12 litters (IOI animals) divided into 3 groups placed in different environmental temperature conditions $\left(\mathrm{I} \mathrm{I}^{\circ} \mathrm{C}, 18^{\circ} \mathrm{C}, 28^{\circ} \mathrm{C}\right)$ from the moment of farrowing. The piglets were suckling ad libitum.

Measurements of weight and rectal temperature were made for each piglet every 5 minutes, then at longer intervals until the $5^{\text {th }}$ day.

$2 \mathrm{ml}$ blood samples were taken off the animals 7 times from the and hour on. The levels of reducing sugars, glucose and fructose were estimated from the samples.

Growth rate was the highest at $28^{\circ} \mathrm{C}$ and the lowest at $I I^{\circ} \mathrm{C}$. Mortality rate reached a maximum at $I I^{\circ} \mathrm{C}$. In a parallel way, the lower the environmental temperature was, the longer the decrease in rectal temperature noticed immediately after birth $\left(39\right.$ to $34.5^{\circ} \mathrm{C}$ on the average) was : $30 \mathrm{mn}$ at $28^{\circ} \mathrm{C}$ v. 2 hours at ${ }^{\circ}{ }^{\circ} \mathrm{C}$.

The levels of reducing sugars and blood glucose were high at birth ( $\mathrm{I} .2$ and $0.8 \mathrm{~g}$ per liter respectively). The higher the environmental temperature, the faster they reached a minimum.

The fructose rate decreased within the first 2 days of life and remained low though constant.

\section{RÉFÉRENCES BIBLIOGRAPHIQUES}

Alexander G., MCCance R. A., 1958. Temperature regulation in the new-born lamb. I. Changes in rectal temperature within the first six hours of life. Austr. J. Agric. Res., 9, 339-347.

Curtis S. E., Heidenreich C. J., Foley C. W., 1966. Carbohydrate assimilation and utilization by new-born pigs. J. Anim. Sci., 25, 655-662.

DAwES G.S., Motr J. C., 1959. The increase in oxygen consumption of the lamb after birth. J. Physiol. 146, 295-313.

DunCAN D. B., I 955. Multiple range and multiple F tests. Biometrics, 11, 1-42.

Elneil H., MCCANCE R. A., 1965. The effect of environmental temperature on the composition and carbohydrate metabolism of new-born pig. J. Physiol., 179, 278-284.

Farquhar J. W., 1954. Control of the blood sugar level in the neonatal period. Arch. Dis. Child., 29, 519529 .

Goodwin R. F. W., 1952. Fotal fructose in various mammals. Nature, London, 170, 750.

Hahn P., Koldovsky O., Ig6r $a$ ). The effect of individual nutrients on growth and carbohydrate formation in rats of differents ages. Physiol. Bohemoslovenica., 10, 481-487.

HahN P., Koldovsky O., MrlichaR M., Novak M., I961 b). Interrelationship between fat and sugar metabolism in infant rats. Vature, London, 192, 1296.

Hain P., Koldovsky O., 1963. Carbohydrate utilization in suckling rats. Physiol. Bohemoslovenica., $12,453-457$.

Holub A., Formax Z., Jezkoya D., 1957. Development of chemical thermo-regulation in piglets. Nature London, 180, $858-859$.

Hopkins J. A., 1928. An economic study of the hog enterprise in Humbolt country. Iora Agr. Exp. Sta., Bull., 255.

Hugett A. S. G., Nixon D. A., r 957. Use of glucose oxidase, peroxidase and O-dianisidine in determination of blood and urinary glucose. I ancet, 368-370.

Ingram D. L., Slebodzinski A., 1965. Oxygene consumption and thyroïd gland activity during adaptation to high ambiant temperature in young pigs. Res. Vet. Sci., 6, 522-530.

JENSEN A. H., I 964. Symposium on environment and facilities : Environment and facilities in swine production. J. Anim. Sci., 23, I 185-1 I 96. 
Kayser C., I963. Physiologie 11I. Les grandes fonctions. Éd. Méd., Flammarion, Paris.

Kidder D. E., Manners M. J., McCrea M. R., Weaver B. M. Q., I963. Fructose utilization in the piglet. Res. Veter. Sci., 4, I $45^{-1} 50$.

Legault C., Canonge J. C., I965. Essai d'application d'un test de convergence de droites de régression à la croissance des porcelets. Ann. Zootech., 14, 285-298.

Leroy A. M., 1953. Variation des besoins alimentaires des porcs en fonction de la saison. Ann. Zoolech., 2, $1-9$.

Loiseleur J., I963. Techniques de laboratoire. Tome II. Chimie clinique. Nasson (ie. éd. Paris.

Lucas I. A. M., I954. Some further observations upon pigs reared in cold pens. J. Agric. Sci., 44, 369376.

Michance K. A., Widdowsox E. M., 1959. The effect of lowering the ambiant temperature on the metabolism of the new-born pig. J. Physiol., 147, 124-134.

McLagan J. R., Thomson W., i950. Fffective temperature as a measure of envirommental conditions for pigs. J. Agric. Sci, 40, 367-374.

Markovic P., I963. Thermoregulation in young cattle. Zivocisna Vyroba., 6, 1sı-216.

Marks V., Lloyn K., I963. Preservation of blood samples for glucose analysis by glucose oxidase. Clin. Chim. Acta., 8, 326.

Morrill C. C., I952. Studies on baby pig mortality. X Influcnce of environmental temperature on fasting new-born pigs. Amer. J. Vet. Res., 13, $3^{22-324 .}$

Mount I. E., I959. The metabolic rate of the new-born pig in relation to environmental temperature and to age. J. Physiol., 147, $333^{-345 .}$

Mount L. E., r96r. Metabolic rate and body temperature in the new-born pig. Agric. Res. Council Insi. Anim. Physiol., 7, n $^{\circ} 3 \mathbf{3} 3$.

Newland H. W., McMillen W. N., Reineke E. P., I952. Temperature adaptation in the baby pig J. Anim. Sci., 11, I I 8-I 33 .

Novak M., Melichar V., Hahn P., Koldovsky O., I965. Release of free fatty acids from adipose tissue obtained from new-born infants. J. Lipid. Res., 6, 91-95.

RoE J. H., I 934. A colorimetric method for the determination of fructose in blood and urine. J. Biol. Chem. 107, $15-22$.

Runcis A. Jr., 1956. The effect of low temperature on the rearing of pig. Zivotnovodslvo, 2, 76-77.

Salmon-LegagneuR E., Guéguen I., rg62. Évolution de la composition chimique du colostrum chez la Truie. Ann. Zootech., 12, 299-317.

Sinelley H. J., Neligan G. A., I966. The foetus and new-born. Recent research on neonatal hypoglycemia. Brit. Med. Bull., 22, 34-39.

Smitr C. A., 1959. The physiology of the neze-born. 3 rd Ed. Thomas and Co, Springfield I I I.

Somogyi M., 1945. Determination of blood sugar. J. Biol. Chem., 160, 69-73.

Watson D., 1962. Enzymic determination of glucose and easily hydrolyzable glucose esters in blood. Anal. Biochem., 3, 13I-144.

Wallach D. P., Newland H. W., McMillen W. N., 1948. Some studies on temperature adaptation in the baby pig. Mich. Agr. Exp. Sta. Quart. Bull., arr.

Zukov F. A., rg62. Result of rearing winter litters in non-heated piggeries. Sovet. Zootekk., 9, 94-95. Biochem., 3, I31-I 44 . 